\title{
Estructura poblacional y hábitat de un árbol tropical con frutos comestibles, Annona purpurea (Annonaceae), en el occidente de México
}

\author{
Citlally Topete-Corona ${ }^{1}$, Ramón Cuevas-Guzmán ${ }^{1 *}$, Enrique V. Sánchez-Rodríguez ${ }^{1}$, Arturo
} Moreno-Hernández ${ }^{2}$, José Guadalupe Morales-Arias ${ }^{1} \&$ Nora M. Núñez-López ${ }^{1}$

1. Departamento de Ecología y Recursos Naturales, Centro Universitario de la Costa Sur, Universidad de Guadalajara. Av. Independencia Nacional 151, Autlán de Navarro, Jalisco, México. C.P. 48900; citlally.topete@cucsur.udg.mx, rcuevas@cucsur.udg.mx, valentes@cucsur.udg.mx, jose.moralesa@cucsur.udg.mx, nnunez@cucsur.udg.mx

2. Departamento de Producción Agrícola, Centro Universitario de la Costa Sur, Universidad de Guadalajara. Av. Independencia Nacional 151, Autlán de Navarro, Jalisco, México. C.P. 48900; amoreno@cucsur.udg.mx

* Correspondencia

Recibido 07-VI-2020. Corregido 11-VIII-2020. Aceptado 19-VIII-2020.

\begin{abstract}
Population structure and habitat of a tropical tree with edible fruits, Annona purpurea (Annonaceae), in Western Mexico. Introduction: Annona purpurea is a Mesoamerican tree, distributed from the Pacific and Atlantic slopes of Mexico to South America. Different parts of the plant are of utility and the fruits are edible. Objective: To describe the population structure, habitat and environmental factors that influence the distribution and abundance of $A$. purpurea in Western Mexico. Methods: From April to November 2015, 24 sampling units were established, each of which were $500 \mathrm{~m}^{2}$ in area and had a presence of A. purpurea. At each site, all woody species with a diameter at breast height (dap) $\geq 2.5 \mathrm{~cm}$ were measured and identified, and environmental, geographic and climatic information recorded. Information regarding the regeneration of the species was obtained. The structure of $A$. purpurea was estimated and its relationships with environmental variables examined. The Whittaker association index was determined using the importance value index matrix (IVI). With the matrix of environmental variables and that of IVI, a Canonical Correspondence Analysis was performed to determine the influence of environmental variables on the species and to visualize the distribution of the species in multidimensional space. Results: A. purpurea presented 1108 stems in 1.2 ha, with 85 $\%$ of these concentrated in the first three diametric categories. Its density presented a positive relationship with the presence of stumps, while the basal area and IVI were similarly related to the incidence of fire. There was little or no regeneration of $A$. purpurea under its canopy. The highest IVI in the community were found for $A$. purpurea, Tabebuia rosea, Quercus magnoliifolia and Enterolobium cyclocarpum, and the similarity profiles test separated A. purpurea and Guazuma ulmifolia as a distinct group. The environmental variables with the greatest influence on the distribution and abundance of $A$. purpurea were annual precipitation, fire incidence, elevation, mean annual temperature, stoniness and livestock. Conclusion: A. purpurea presents the greatest structural attributes in the community, its populations are favored in places with greater precipitation and temperature, with little stoniness and with incidence of disturbances by fire and livestock that generate large gaps and its greatest association is with G. ulmifolia.
\end{abstract}

Key words: cabeza de negro; fire; livestock; tropical subdeciduous forest; secondary vegetation.

Topete-Corona, C., Cuevas-Guzmán, R., Sánchez-Rodríguez, E.V., Moreno-Hernández, A., Morales-Arias, J.G., \& Núñez-López, N.M. (2020). Estructura poblacional y hábitat de un árbol tropical con frutos comestibles, Annona purpurea (Annonaceae), en el occidente de México. Revista de Biología Tropical, 68(4), 1171-1184. 
Annona es un género con 160 especies, 156 se conocen para el Neotrópico (Maas et al., 2019), de ellas, 18 se han registrado para México (De la Cruz, Castro-Montero, González-Esquinca, 2016), algunas descritas recientemente (Jiménez-Ramírez \& Soto-Núñez, 2015). La importancia de las anonas por sus frutos comestibles se tiene ampliamente documentada (Standley, 1920; Luna-Cázares \& González-Esquinca, 2015), por lo que algunas especies se cultivan para atender la demanda de frutos en los mercados nacionales e internacionales y otras se recolectan de poblaciones silvestres, entre ellas A. purpurea (AndresAgustín, 2015).

Annona purpurea se conoce con más de 60 nombre locales (Luna-Cázares \& GonzálezEsquinca, 2015), estando entre los más generalizados "cabeza de negro", "chinguya" y "sincuya", haciendo alusión al fruto, el cual es cosechado y vendido en los mercados locales (Standley, 1920, Luna-Cázares, \& GonzálezEsquinca, 2015; Topete-Corona, 2018), por lo que ha sido considerada una especie de interés frutícola (Standley, 1920; Andres-Agustín, 2015). Se trata de una especie Mesoamérica, con una distribución desde Nayarit y Veracruz, en México, hasta Venezuela, Colombia y Ecuador (Topete-Corona, 2018), en bosques tropicales perennifolios a caducifolios y áreas abiertas dentro de ellos (Luna-Cázares \& González-Esquinca, 2015) y en bosques de encino (Topete-Corona, 2018).

Los pobladores mesoamericanos han utilizado las hojas, tallos, raíces, frutos y semillas de la especie, con fines medicinales, como insecticida y antibacterial (Standley, 1920; LunaCázares \& González-Esquinca, 2008). Los estudios de las propiedades químicas de estas estructuras, revelan la presencia de sustancias con potencial como anticancerígenas, hipoglucémicas, antibacteriales y para la producción de insecticidas y biocombustibles (Luna-Cázares \& González-Esquinca, 2008; Vidal-Lezama, Villegas-Monter, Vaquera-Huerta, RobledoPaz, \& Martínez-Palacios, 2019).

Se considera que en algunas regiones de México las poblaciones de la especie están disminuyendo, debido principalmente a la alteración de su hábitat (Vidal-Lezama et al., 2019). A pesar de lo anterior no se conocen investigaciones sobre la estructura de las poblaciones y el hábitat de $A$. purpurea, por lo que el objetivo de la investigación es describir la estructura poblacional, el hábitat y los factores ambientales que influyen sobre la distribución y abundancia de la especie en el occidente de México.

\section{MATERIALES Y MÉTODOS}

Área de estudio: La investigación se realizó en la zona tropical de los estados de Jalisco y Colima en el occidente de México (104 $03^{\circ}$ '$104^{\circ} 54^{\prime} \mathrm{N} \& 19^{\circ} 20^{\prime}-19^{\circ} 46^{\prime} \mathrm{W}$ ), a una elevación de 300-800 m, con una temperatura media anual de $22.8-25.1^{\circ} \mathrm{C}$ y una precipitación anual de $1346-1480 \mathrm{~mm}$; con un periodo de sequía de 6-8 meses y una concentración del $90 \%$ o más de la precipitación de junio a septiembre (Fernández, Zavala, \& Romero-Fernández, 2010). La vegetación que se registra en el área corresponde con bosque tropical caducifolio y subcaducifolio y, encinares caducifolios (Rzedowski \& McVaugh, 1966).

Registro de datos en campo: De abril a noviembre 2015, se establecieron 24 unidades circulares de $500 \mathrm{~m}^{2}$, a través de un muestreo selectivo de áreas con presencia de $A$. purpurea. Dentro de cada unidad de muestreo se identificaron, midieron y registraron las especies leñosas con diámetro a la altura de pecho (dap) $\geq 2.5 \mathrm{~cm}$. Dentro de cada parcela $\left(500 \mathrm{~m}^{2}\right) \mathrm{se}$ establecieron cinco subparcelas circulares de $5 \mathrm{~m}^{2}$ cada una, una al centro y las otras cuatro hacia los extremos de la parcela, en orientación norte-sur y este-oeste, con la finalidad de evaluar la repoblación de $A$. purpurea, registrando y midiendo los individuos con un dap $<2.5$ $\mathrm{cm}$. Se recolectaron ejemplares de las especies para corroborar las identificaciones y se depositaron en el Herbario ZEA de la Universidad de Guadalajara, como material de referencia de la investigación. Para cada parcela se registró coordenadas, elevación, tipo de vegetación, 
pendiente, exposición, factores de perturbación, entre otros (Tabla 1). Para obtener por localidad la temperatura y la precipitación, se utilizaron datos disponibles en el Atlas Climático Digital de México (Fernández et al., 2010).

Análisis de los datos: Estructura poblacional de A. purpurea: se definió en función de los tallos con dap $\geq 2.5 \mathrm{~cm}$ registrados en las 24 parcelas. Las clases diamétricas se realizaron en función de: $\mathrm{M}=3 \log _{10} \mathrm{~N}$, donde $\mathrm{M}$ = número de clases diamétricas $\mathrm{y} \mathrm{N}=$ número de tallos de A. purpurea. El intervalo de clase fue igual a (diámetro mayor-diámetro menor) / M. Se determinó por parcela la densidad, área basal (AB) y el índice de valor de importancia (IVI) de A. purpurea, este último como la suma de los promedios relativos de densidad y área basal (Curtis \& McIntosh, 1951; Vázquez \& Givnish, 1998; Cuevas, García-Moya, Vázquez-García, \& Núñez-López, 2008).

El análisis de la repoblación se realizó considerando los individuos de A. purpurea con dap $<2.5 \mathrm{~cm}$ registrados en las 24 parcelas,

TABLA 1

Información geográfica y ambiental de las unidades de muestreo de A. purpurea en el occidente de México

TABLE 1

Geographic and environmental information of the sampling units of $A$. purpurea in Western Mexico

\begin{tabular}{|c|c|c|c|c|c|c|c|c|c|c|c|c|c|}
\hline Par & Lat & Lon & Ele & Pen & Exp & Ped & Phs & Inc & Pas & Amu & Toc & Tma & Pan \\
\hline P01 & $19^{\circ} 36^{\prime} 28^{\prime \prime}$ & $104^{\circ} 18^{\prime} 33^{\prime \prime}$ & 650 & 14 & $20^{\circ} \mathrm{NO}$ & 4 & 20 & 0 & 4 & 0 & 2 & 24.0 & 1308 \\
\hline P02 & $19^{\circ} 36^{\prime} 26^{\prime \prime}$ & $104^{\circ} 18^{\prime} 31^{\prime \prime}$ & 650 & 11 & $30^{\circ} \mathrm{NO}$ & 4 & 22 & 0 & 4 & 5 & 4 & 24.0 & 1308 \\
\hline $\mathrm{P} 03$ & $19^{\circ} 36^{\prime} 41^{\prime \prime}$ & $104^{\circ} 18^{\prime} 38^{\prime \prime}$ & 550 & 17 & $40^{\circ} \mathrm{NO}$ & 1 & 35 & 0 & 3 & 1 & 2 & 24.0 & 1304 \\
\hline P04 & $19^{\circ} 40^{\prime} 18^{\prime \prime}$ & $104^{\circ} 25^{\prime} 46.4^{\prime \prime}$ & 662 & 44 & $20^{\circ} \mathrm{NE}$ & 4 & 30 & 4 & 3 & 2 & 4 & 24.1 & 1281 \\
\hline P05 & $19^{\circ} 40^{\prime} 17.7^{\prime \prime}$ & $104^{\circ} 25^{\prime} 57.7$ & 630 & 31 & $35^{\circ} \mathrm{NO}$ & 1 & 20 & 1 & 2 & 0 & 7 & 24.1 & 1281 \\
\hline P06 & $19^{\circ} 27^{\prime} 15.9^{\prime \prime}$ & $104^{\circ} 29^{\prime} 04^{\prime \prime}$ & 603 & 57 & $88^{\circ} \mathrm{NO}$ & 2 & 25 & 4 & 2 & 1 & 0 & 24.2 & 1540 \\
\hline P07 & $19^{\circ} 27^{\prime} 15.2^{\prime \prime}$ & $104^{\circ} 29^{\prime} 8.5^{\prime \prime}$ & 565 & 10 & $40^{\circ} \mathrm{SE}$ & 3 & 30 & 1 & 3 & 1 & 2 & 24.2 & 1540 \\
\hline P08 & $19^{\circ} 31^{\prime} 12.9^{\prime \prime}$ & $104^{\circ} 26^{\prime} 7.7 ”$ & 485 & 5 & $83^{\circ} \mathrm{NE}$ & 1 & 10 & 4 & 4 & 0 & 0 & 25.0 & 1578 \\
\hline P09 & $19^{\circ} 23^{\prime} 39.9^{\prime \prime}$ & $104^{\circ} 27^{\prime} 37.6^{\prime \prime}$ & 639 & 50 & $03^{\circ} \mathrm{SE}$ & 1 & 30 & 4 & 4 & 0 & 1 & 23.9 & 1509 \\
\hline P10 & $19^{\circ} 23^{\prime} 40^{\prime \prime}$ & $104^{\circ} 27^{\prime} 34.7^{\prime \prime}$ & 672 & 28 & $32^{\circ} \mathrm{SE}$ & 2 & 10 & 4 & 4 & 0 & 0 & 23.9 & 1509 \\
\hline P11 & 19॰38'29.7" & $104^{\circ} 54^{\prime} 11.8^{\prime \prime}$ & 354 & 12 & $65^{\circ} \mathrm{NE}$ & 1 & 10 & 4 & 4 & 1 & 2 & 25.1 & 1244 \\
\hline $\mathrm{P} 12$ & $19^{\circ} 35^{\prime} 25.8^{\prime \prime}$ & $104^{\circ} 38^{\prime} 31^{\prime \prime}$ & 439 & 7 & $14^{\circ} \mathrm{SE}$ & 2 & 10 & 4 & 4 & 0 & 0 & 24.7 & 1475 \\
\hline P13 & $19^{\circ} 34^{\prime} 34.7^{\prime \prime}$ & $104^{\circ} 92^{\prime} 10.3^{\prime \prime}$ & 561 & 32 & $58^{\circ} \mathrm{SE}$ & 4 & 7 & 4 & 4 & 0 & 0 & 24.2 & 1470 \\
\hline P14 & $19^{\circ} 34^{\prime} 51.1 "$ & $104^{\circ} 42^{\prime} 11.1^{\prime \prime}$ & 565 & 30 & $86^{\circ} \mathrm{SE}$ & 2 & 10 & 4 & 3 & 2 & 0 & 25.0 & 1278 \\
\hline P15 & $19^{\circ} 34^{\prime} 07.3^{\prime \prime}$ & $104^{\circ} 42^{\prime} 04.5^{\prime \prime}$ & 579 & 18 & $62^{\circ} \mathrm{SE}$ & 1 & 10 & 4 & 4 & 0 & 4 & 25.1 & 1238 \\
\hline P16 & $19^{\circ} 20^{\prime} 22^{\prime \prime}$ & $104^{\circ} 03^{\prime} 58.4^{\prime \prime}$ & 697 & 52 & $15^{\circ} \mathrm{SO}$ & 2 & 15 & 1 & 3 & 1 & 0 & 22.8 & 1331 \\
\hline P17 & $19^{\circ} 20^{\prime} 0.94^{\prime \prime}$ & $104^{\circ} 04^{\prime} 08.8^{\prime \prime}$ & 715 & 22 & $45^{\circ} \mathrm{SO}$ & 2 & 5 & 1 & 3 & 10 & 3 & 23.3 & 1465 \\
\hline P18 & $19^{\circ} 21^{\prime} 25.1^{\prime \prime}$ & $104^{\circ} 05^{\prime} 46^{\prime \prime}$ & 632 & 48 & $52^{\circ} \mathrm{SE}$ & 2 & 10 & 1 & 3 & 4 & 2 & 23.5 & 1291 \\
\hline P19 & $19^{\circ} 21^{\prime} 33.3 "$ & $104^{\circ} 03^{\prime} 48.9^{\prime \prime}$ & 726 & 40 & $80^{\circ} \mathrm{SE}$ & 2 & 7 & 4 & 4 & 0 & 0 & 23.5 & 1478 \\
\hline P20 & $19^{\circ} 44^{\prime} 47.4^{\prime \prime}$ & $104^{\circ} 31^{\prime} 25.9^{\prime \prime}$ & 540 & 40 & $55^{\circ} \mathrm{NO}$ & 2 & 7 & 4 & 4 & 0 & 18 & 24.8 & 1544 \\
\hline P21 & $19^{\circ} 45^{\prime} 14.1^{\prime \prime}$ & $104^{\circ} 31^{\prime} 14.3^{\prime \prime}$ & 546 & 5 & $65^{\circ} \mathrm{NE}$ & 1 & 40 & 1 & 4 & 0 & 2 & 24.7 & 1503 \\
\hline P22 & $19^{\circ} 45^{\prime} 19.3 "$ & $104^{\circ} 31^{\prime} 13.6 "$ & 568 & 8 & $18^{\circ} \mathrm{SE}$ & 1 & 15 & 1 & 4 & 0 & 11 & 24.7 & 1503 \\
\hline P23 & $19^{\circ} 45^{\prime} 1.7 "$ & $104^{\circ} 31^{\prime} 4.9^{\prime \prime}$ & 579 & 30 & $65 \mathrm{SE}$ & 4 & 10 & 1 & 4 & 0 & 2 & 24.7 & 1503 \\
\hline P24 & $19^{\circ} 42^{\prime} 34.4^{\prime \prime}$ & $104^{\circ} 32^{\prime} 37^{\prime \prime}$ & 429 & 16 & $60^{\circ} \mathrm{SE}$ & 1 & 20 & 4 & 4 & 0 & 2 & 25.1 & 1603 \\
\hline
\end{tabular}

Par $=$ parcela, Lat $=$ latitud, Lon $=$ longitud, Ele $=$ elevación en m s.n.m., Pen $=$ Pendiente $(\%)$, Exp $=$ exposición, Ped $=$ pedregosidad, $\mathrm{Phs}=$ profundidad del horizonte superficial $(\mathrm{cm}), \mathrm{Inc}=$ incendios, $\mathrm{Pas}=$ pastoreo, $\mathrm{Amu}=$ árboles muertos, Toc $=$ tocones, $\mathrm{Tma}=$ temperatura media anual $\left({ }^{\circ} \mathrm{C}\right)$, Pan $=$ precipitación anual $(\mathrm{mm})$.

Par $=$ plot, Lat $=$ latitude, Lon $=$ longitude, Ele $=$ elevation in m.a.s.1., Pen $=$ slope $(\%)$, Exp $=$ exposition, Ped $=$ stoniness, $\mathrm{Phs}=$ depth superficial horizon $(\mathrm{cm})$, Inc $=$ fires, Pas $=$ livestock, $\mathrm{Amu}=$ dead trees, $\mathrm{Toc}=$ stumps, Tma $=$ average annual temperature $\left({ }^{\circ} \mathrm{C}\right)$, Pan $=$ annual precipitation $(\mathrm{mm})$. 
realizando las sumas de los individuos registrados en las subparcelas de cada parcela. Se realizaron correlaciones entre los atributos estructurales de $A$. purpurea y variables ambientales en busca de asociación entre los parámetros. Para las variables cuantitativas se aplicó la correlación de Pearson, para las cualitativas (Ped, Pas e Inc) la de Spearman (Balzarini et al., 2008). Las variables ambientales incluidas fueron la elevación (Ele), registrada en el centro de cada parcela con un GPS Garmin Etrex, la pendiente (Pen), registrada con una pistola Haga, la exposición (Exp), tomada con una brújula Brunton, la pedregosidad (Ped), incidencia de incendios (Inc) y pastoreo (Pas), con base en los criterios establecidos en Olvera, Moreno y Figueroa (1996), la profundidad del horizonte superficial (Phs), registrada en cada subparcela de $5 \mathrm{~m}^{2}$ y promediada para toda la parcela, se midió en $\mathrm{cm}$ con una regla graduada, tocones (Toc), se contabilizaron al interior de la parcela de 500 $\mathrm{m}^{2}$, considerando únicamente aquellos $\geq 5 \mathrm{~cm}$ de diámetro a los $30 \mathrm{~cm}$ de altura, con señales claras de haber sido cortados por el hombre, árboles muertos (Amu), se contabilizaron al interior de la parcela, incluyendo aquellos en pie y caídos que no habían iniciado su descomposición y con un dap $\geq 10 \mathrm{~cm}$. Los análisis de los datos se realizaron utilizando el software InfoStat (Di Rienzo, Casanoves, Balzarini, Gonzalez, Tablada, \& Robledo 2019), PC-ORD v7 (McCune \& Mefford, 2011) y Primer v7 (Clarke \& Gorley, 2015).

Especies asociadas con A. purpurea $y$ estructura de la comunidad: Para el análisis de asociación de especies, y determinar con cuáles $A$. purpurea tiene sus mayores relaciones y con cuáles se agrupa, se utilizó la matriz de IVI, considerando sólo las especies que presentaron un índice $\geq 10 \%$ en una o más parcelas. Las especies fueron estandarizadas dividiendo el valor de cada una de ellas por su total y multiplicándolo por 100, seguido por un cálculo de la semejanza Bray-Curtis entre pares de especies, lo que se conoce como índice de asociación de especies de Whittaker (Clarke, Gorley, Somerfield, \& Warwick, 2014). Con la matriz de semejanza de las especies se realizó un análisis de agrupamiento, con grupo promedio como método de unión de grupos, acompañado de una prueba de perfiles de semejanza (SIMPROF) tipo 2 y 3 . La prueba tipo 2 trabaja con la hipótesis nula de la no existencia de asociaciones entre las especies y la tipo 3 con la no existencia de diferencias entre los grupos de especies, teniendo como prueba estadística a $\pi$ (pi), la cual es una prueba de permutaciones, para la cual se puede encontrar más información en Clarke et al. (2014). Los resultados se expresan a través de un dendrograma, en el cual los grupos estadísticamente disimiles se diferencian por una línea continua.

Se determinó la riqueza de especies, densidades, AB e IVI para las especies por parcela (Curtis \& McIntosh, 1951; Vázquez-García \& Givnish, 1998; Cuevas et al., 2008). Se integró una matriz de IVI con las 23 que contribuyeron con al menos el $10 \%$ en una o más parcelas. La matriz de variables ambientales incluyó la Ele, Pen, Exp, Ped, Phs, Inc, Pas, Amu, Toc, Tma, Pan (Tabla 1). Las variables cualitativas como la Ped, Inc y Pas, fueron convertidas en variables auxiliares (Dummy) y se trataron como cuantitativas. La variable Exp fue transformada siguiendo el criterio de Beers, Dress y Wensel (1966). Quitando las variables con autocorrelaciónes significativas (McCune \& Mefford, 2011) e incluyendo las variables cualitativas transformadas a dummy, la matriz se integró por 11 variables ambientales. Utilizando las dos matrices se hizo un Análisis de Correspondencia Canónica (ACC) seleccionando las opciones: los resultados de los ejes centrados y estandarizados a una unidad de varianza, los ejes escalados para representar la optimización de las especies, los resultados finales de la ordenación en el gráfico representando combinaciones lineales de las variables ambientales, y la hipótesis nula de la no existencia de relación entre la matriz de las especies y la de variables ambientales, con 999 iteraciones (McCune \& Mefford, 2011). La prueba estadística para aceptar o rechazar la hipótesis nula se realiza a través de una prueba de permutaciones (prueba de Monte Carlo), 
la cual muestra como los valores propios observados (eigenvalores) y, las correlaciones entre las especies y el ambiente comparan con los de las corridas aleatorias y determinando un valor de "p" (McCune \& Grace, 2002).

\section{RESULTADOS}

Estructura poblacional de $A$. purpurea y su relación con variables ambientales: $\mathrm{Se}$ registraron 402 individuos de $A$. purpurea con 1108 tallos con un diámetro $\geq 2.5 \mathrm{~cm}$ en una superficie de 1.2 ha. La altura promedio de los individuos fue de $9.34 \pm 3.64 \mathrm{~m}$, registrándose
34 individuos con alturas entre 15-18 m. El 76 $\%$ de los individuos presentó entre uno y tres tallos y sólo el $24 \%$ tuvo más de tres, encontrándose 24 individuos con 10-15 tallos. El diámetro promedio de los tallos fue de $9.11 \pm 5.96$ cm. La densidad de tallos de A. purpurea por parcela osciló entre 13-105 con una media de $46.17 \pm 7.27$, mientras que el $\mathrm{AB}$ fue de 0.077 $0.72 \mathrm{~m}^{2}$ con una media de $0.0093 \pm 0.014$ y los IVI estuvieron entre $15.67-83.43 \%$ con una media de $48.31 \pm 21.54$ por parcela (Tabla 2 ). La densidad de $A$. purpurea representó el 49 $\%$ (1 108 tallos $1.2 \mathrm{ha}^{-1}$ ) y el AB el $38.31 \%$ $\left(10.30 \mathrm{~m}^{2} 1.2 \mathrm{ha}^{-1}\right)$ de la comunidad.

TABLA 2

Atributos estructurales de $A$. purpurea por unidad de muestreo y atributos estructurales de la comunidad

TABLE 2

Structural attributes of $A$. purpurea by sampling unit and structural attributes of the community

\begin{tabular}{ccccccccccc} 
Par & Den AP & Tal AP & Aba AP & IVI AP & Reg AP & Fam & Gén & Spp & Aba Com & Tal Com \\
P01 & 16 & 18 & 0.1546 & 22.53 & 0 & 13 & 15 & 16 & 1.58 & 51 \\
P02 & 16 & 16 & 0.0769 & 13.38 & 0 & 14 & 15 & 17 & 1.82 & 71 \\
P03 & 22 & 27 & 0.2585 & 23.66 & 0 & 16 & 19 & 22 & 1.17 & 107 \\
P04 & 40 & 105 & 0.7100 & 77.49 & 6 & 7 & 7 & 7 & 1.08 & 118 \\
P05 & 14 & 50 & 0.1771 & 52.05 & 1 & 6 & 6 & 6 & 0.80 & 61 \\
P06 & 8 & 39 & 0.3883 & 50.2 & 1 & 10 & 13 & 13 & 0.63 & 101 \\
P07 & 15 & 77 & 0.5595 & 63.24 & 1 & 12 & 14 & 15 & 0.80 & 136 \\
P08 & 16 & 53 & 0.4020 & 55.87 & 0 & 7 & 7 & 7 & 0.76 & 90 \\
P09 & 20 & 47 & 0.6924 & 56.31 & 1 & 12 & 16 & 17 & 0.99 & 110 \\
P10 & 10 & 28 & 0.3817 & 43.01 & 2 & 4 & 5 & 5 & 1.23 & 51 \\
P11 & 8 & 27 & 0.4220 & 65.7 & 1 & 6 & 7 & 7 & 0.60 & 44 \\
P12 & 11 & 37 & 0.6032 & 39.3 & 5 & 11 & 14 & 15 & 3.19 & 62 \\
P13 & 11 & 24 & 0.3250 & 29.31 & 1 & 15 & 22 & 24 & 0.97 & 95 \\
P14 & 3 & 13 & 0.2903 & 26.09 & 3 & 14 & 19 & 21 & 0.77 & 89 \\
P15 & 15 & 66 & 0.7179 & 72.56 & 2 & 7 & 8 & 8 & 1.05 & 86 \\
P16 & 14 & 42 & 0.1108 & 24.48 & 0 & 10 & 12 & 17 & 0.57 & 142 \\
P17 & 11 & 80 & 0.3460 & 33.78 & 0 & 16 & 20 & 23 & 1.97 & 160 \\
P18 & 9 & 33 & 0.3108 & 22.74 & 0 & 22 & 23 & 26 & 1.31 & 151 \\
P19 & 13 & 38 & 0.7084 & 56.85 & 3 & 10 & 12 & 12 & 0.97 & 93 \\
P20 & 53 & 79 & 0.3764 & 61.82 & 2 & 8 & 10 & 11 & 0.77 & 106 \\
P21 & 8 & 18 & 0.3504 & 52.45 & 0 & 6 & 7 & 8 & 0.86 & 28 \\
P22 & 24 & 103 & 0.7411 & 92.16 & 0 & 5 & 5 & 6 & 0.77 & 117 \\
P23 & 37 & 68 & 0.4720 & 38.89 & 0 & 10 & 14 & 15 & 1.27 & 168 \\
P24 & 8 & 20 & 0.7294 & 85.67 & 0 & 2 & 2 & 2 & 0.96 & 21 \\
\hline
\end{tabular}

Par $=$ parcela, Den AP = densidad de individuos de $A$. purpurea, $\mathrm{Tal}$ AP $=$ densidad de tallos de $A$. purpurea, $\mathrm{Aba} \mathrm{AP}=$ área basal de $A$. purpurea $\left(\mathrm{m}^{2}\right)$, IVI AP = índice de valor de importancia de $A$. purpurea $(\%)$, Reg AP = regeneración de $A$. purpurea, $\mathrm{Fam}=$ familias, $\mathrm{Gén}=$ géneros, $\mathrm{Spp}=$ especies, $\mathrm{Aba} \mathrm{Com}=$ área basal de la comunidad $\left(\mathrm{m}^{2}\right), \mathrm{Tal} \mathrm{Com}=\mathrm{densidad}$ de tallos en la comunidad.

Par $=$ plot, Den AP $=$ density of $A$. purpurea individuals, Tal AP $=$ stem density of $A$. purpurea, Aba AP $=$ basal area of $A$. purpurea $\left(\mathrm{m}^{2}\right)$, IVI AP = importance value index of $A$. purpurea $(\%)$, Reg AP = regeneration of $A$. purpurea, Fam $=$ families, $\mathrm{Gen}=$ genera, $\mathrm{Spp}=$ species, $\mathrm{Aba} \mathrm{Com}=$ basal area of the community $\left(\mathrm{m}^{2}\right)$, Tal Com $=$ stem density in the community. 
La estructura diamétrica de $A$. purpurea presentó una forma de "J" invertida, con una mayor concentración de tallos en las categorías diamétricas inferiores y una disminución hacia las superiores, con el $85 \%$ de los tallos en las tres primeras categorías (Fig. 1). La densidad de tallos mostró una correlación positiva y significativa con la presencia de Toc $(\mathrm{r}=0.53$, $\mathrm{P}=0.01$ ), el $\mathrm{AB}$ y el IVI con la incidencia de Inc $(\mathrm{r}=0.55$ y $\mathrm{r}=0.50, \mathrm{P}=0.005$ y $\mathrm{P}=0.01$, respectivamente) y el IVI con la Tma $(\mathrm{r}=0.5 \mathrm{y}$ $\mathrm{P}=0.01)$ y negativa con la Ped $(\mathrm{r}=-0.48, \mathrm{P}=$ 0.029). La regeneración fue escasa, en 11 parcelas no se registraron individuos y solo en una se encontraron seis. Una correlación positiva y significativa se obtuvo entre la incidencia de Inc y la regeneración $(\mathrm{r}=0.70, \mathrm{P}=0.001)$.
Estructura de la comunidad y hábitat de $A$. purpurea: Se registraron 47 familias, 87 géneros y 109 especies de plantas leñosas con un dap $\geq 2.5 \mathrm{~cm}$ en $1.2 \mathrm{ha}$. La riqueza de familias osciló de 2-22 por parcela, la de géneros de 2-23, la de especies de 2-26 (Tabla 2). Las familias con mayor riqueza de especies fueron: Fabaceae (17), Euphorbiaceae (8) y Moraceae, Rubiaceae y Salicaceae (6 cada una). Los géneros con más especies fueron Ficus (4), Casearia, Randia y Vachellia (3 cada uno). El AB por parcela osciló de 0.57-3.19 $\mathrm{m}^{2}$ y la densidad de 21-168 tallos (Tabla 2). Los mayores IVI promedio los tuvieron A. purpurea (48.31\%), Tabebuia rosea (3.96 \%), Quercus magnoliifolia (3.79\%), Enterolobium cyclocarpum (3.42 \%), Lonchocarpus salvadorensis (2.97 \%),

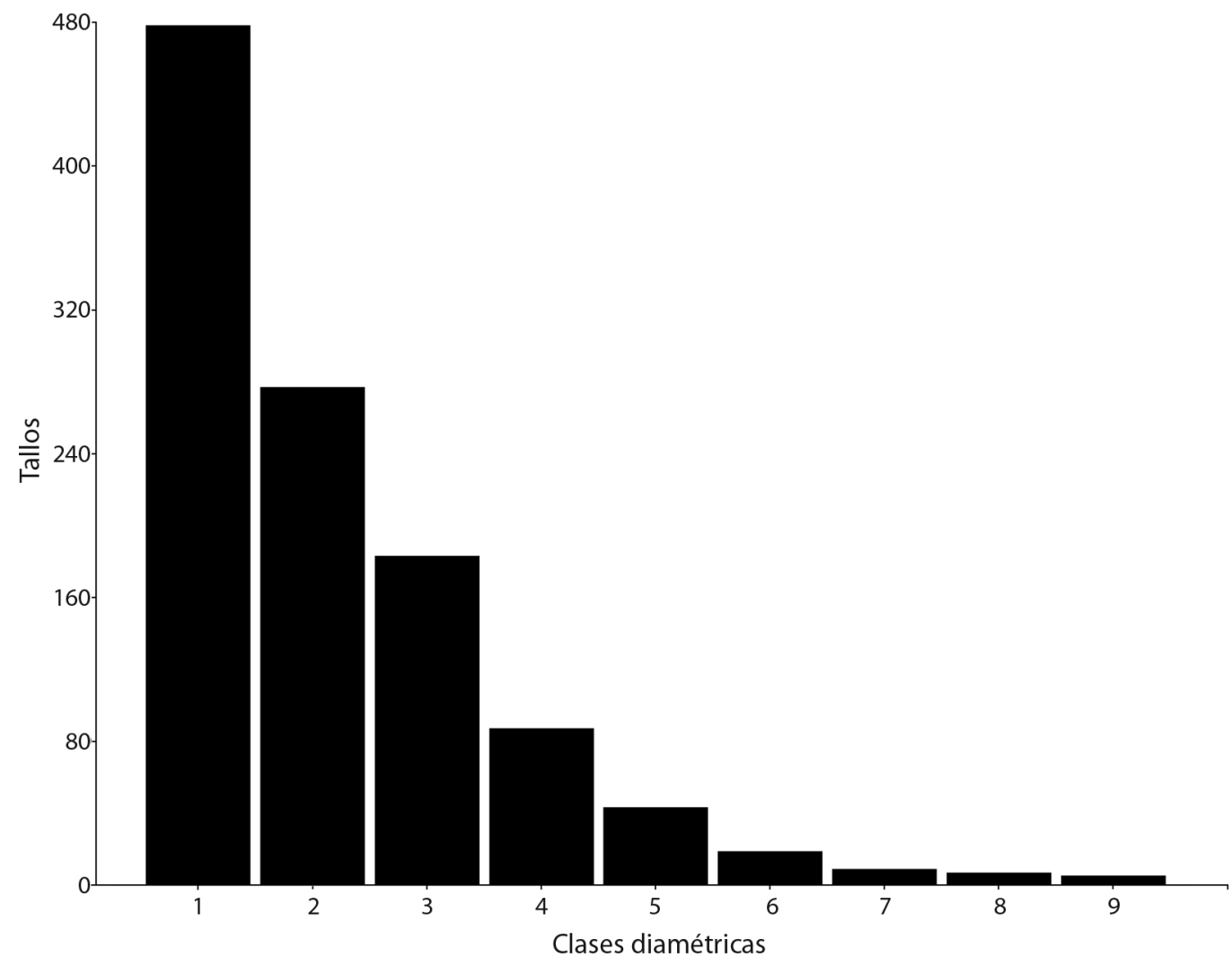

Fig. 1. Estructura diamétrica de A. purpurea. La clase $1=2.50-6.52,2=6.52-10.54,3=10.54-14.57,4=14.57-18.59,5=$ $18.59-22.61,6=22.61-26.63,7=26.63-30.66,8=30.66-34.68,9=34.68-38.70$.

Fig. 1. Diametric structure of $A$. purpurea. Class $1=2.50-6.52,2=6.52-10.54,3=10.54-14.57,4=14.57-18.59,5=18.59$ $22.61,6=22.61-26.63,7=26.63-30.66,8=30.66-34.68,9=34.68-38.70$. 


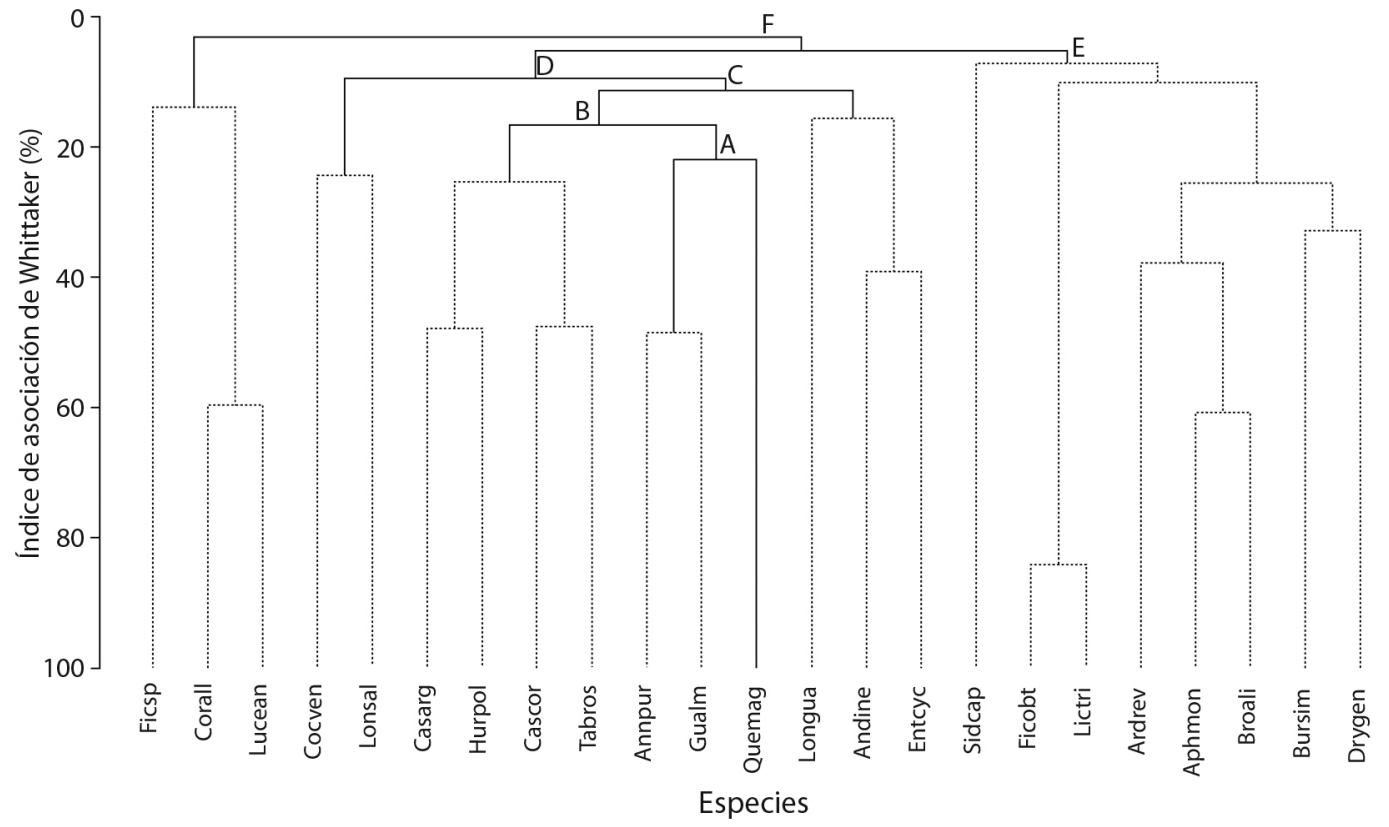

Fig. 2. Dendrograma de las 21 especies más importantes por su IVI. Ficsp = Ficus sp., Corall = Cordia alliodora, Luecan = Luehea candida, Cocven $=$ Coccoloba venosa, Lonsal $=$ Lonchocarpus salvadorensis, Casarg $=$ Casearia arguta, Hurpol $=$ Hura polyandra, Cascor $=$ Casearia corymbosa, Tabros $=$ Tabebuia rosea, Annpur $=$ Annona purpurea, Guaulm $=$ Guazuma ulmifolia, Quemag = Quercus magnoliifolia, Longua $=$ Lonchocarpus guatemalensis, Andine $=$ Andira inermis, Entcyc $=$ Enterolobium cyclocarpum, Sidcap $=$ Sideroxylon capiri, Ficobt $=$ Ficus obtusifolia, Lictri $=$ Licaria triandra, Ardrev $=$ Ardisia revoluta, Aphmon $=$ Aphananthe monoica, Broali $=$ Brosimum alicastrum, Bursim $=$ Bursera simaruba, Drygen $=$ Drypetes gentryi. Líneas continuas indican grupos de especies estadísticamente diferentes. Las letras indican las diferencias estadísticas entre los grupos: $\mathrm{A} \pi=7.37 \mathrm{p}=0.02, \mathrm{~B} \pi=5.36 \mathrm{p}=0.002, \mathrm{C} \pi=4.24 \mathrm{p}=0.001, \mathrm{D} \pi=3.27 \mathrm{p}=0.006, \mathrm{E} \pi=$ $2.38 \mathrm{p}=0.003, \mathrm{~F} \pi=2.2 \mathrm{p}=0.003$.

Fig. 2. Dendrogram of the 21 most important species for their IVI. Ficsp $=$ Ficus sp., Corall = Cordia alliodora, Luecan $=$ Luehea candida, Cocven $=$ Coccoloba venosa, Lonsal $=$ Lonchocarpus salvadorensis, Casarg $=$ Casearia arguta, Hurpol $=$ Hura polyandra, Cascor $=$ Casearia corymbosa, Tabros $=$ Tabebuia rosea, Annpur $=$ Annona purpurea, Guaulm $=$ Guazuma ulmifolia, Quemag = Quercus magnoliifolia, Longua $=$ Lonchocarpus guatemalensis, Andine $=$ Andira inermis, Entcyc $=$ Enterolobium cyclocarpum, Sidcap $=$ Sideroxylon capiri, Ficobt $=$ Ficus obtusifolia, Lictri $=$ Licaria triandra, Ardrev $=$ Ardisia revoluta, Aphmon $=$ Aphananthe monoica, Broali $=$ Brosimum alicastrum, Bursim $=$ Bursera simaruba, Drygen $=$ Drypetes gentryi. Solid lines indicate statistically different groups of species. Letters indicate statistical differences between groups: $\mathrm{A} \pi=7.37 \mathrm{p}=0.02, \mathrm{~B} \pi=5.36 \mathrm{p}=0.002, \mathrm{C} \pi=4.24 \mathrm{p}=0.001, \mathrm{D} \pi=3.27 \mathrm{p}=0.006, \mathrm{E} \pi=2.38 \mathrm{p}=0.003, \mathrm{~F} \pi$ $=2.2 \mathrm{p}=0.003$.

Casearia arguta (2.72\%) y Guazuma ulmifolia (2.67\%). La prueba SIMPROF, mostró seis grupos de especies estadísticamente diferentes (Fig. 2). Annona purpurea tuvo sus mayores índices de asociación con G. ulmifolia (48.58 $\%)$, T. rosea (34.63\%), C. arguta (34.41\%), E. cyclocarpum $(29.17 \%)$ y $Q$. magnoliifolia (29.13\%), formando con G. ulmifolia un grupo estadísticamente diferente a los demás (Fig. 2). Ambas especies compartieron el $58 \%$ de las unidades de muestreo con IVI altos.
El ACC obtuvo una inercia total de 7.57, con una varianza extraída en los tres primeros ejes de $28.1 \%$. El eje 1 extrajo el $10.7 \%$, el 2 el $9.4 \%$ y 3 el $8.1 \%$. Los coeficientes canónicos estandarizados con mayor contribución al eje 1 fueron los Inc_1 e Inc_4 y el Pas_3; para el eje 2 la Ele, Inc_4 y la Ped_2 y para el 3, Ped_2, Ped_4 y el Pas_4 (Tabla 3). La prueba de Monte Carlo para los valores propios de los ejes obtuvo una $\mathrm{p}=0.002$ y para la correlación entre la matriz de las especies 


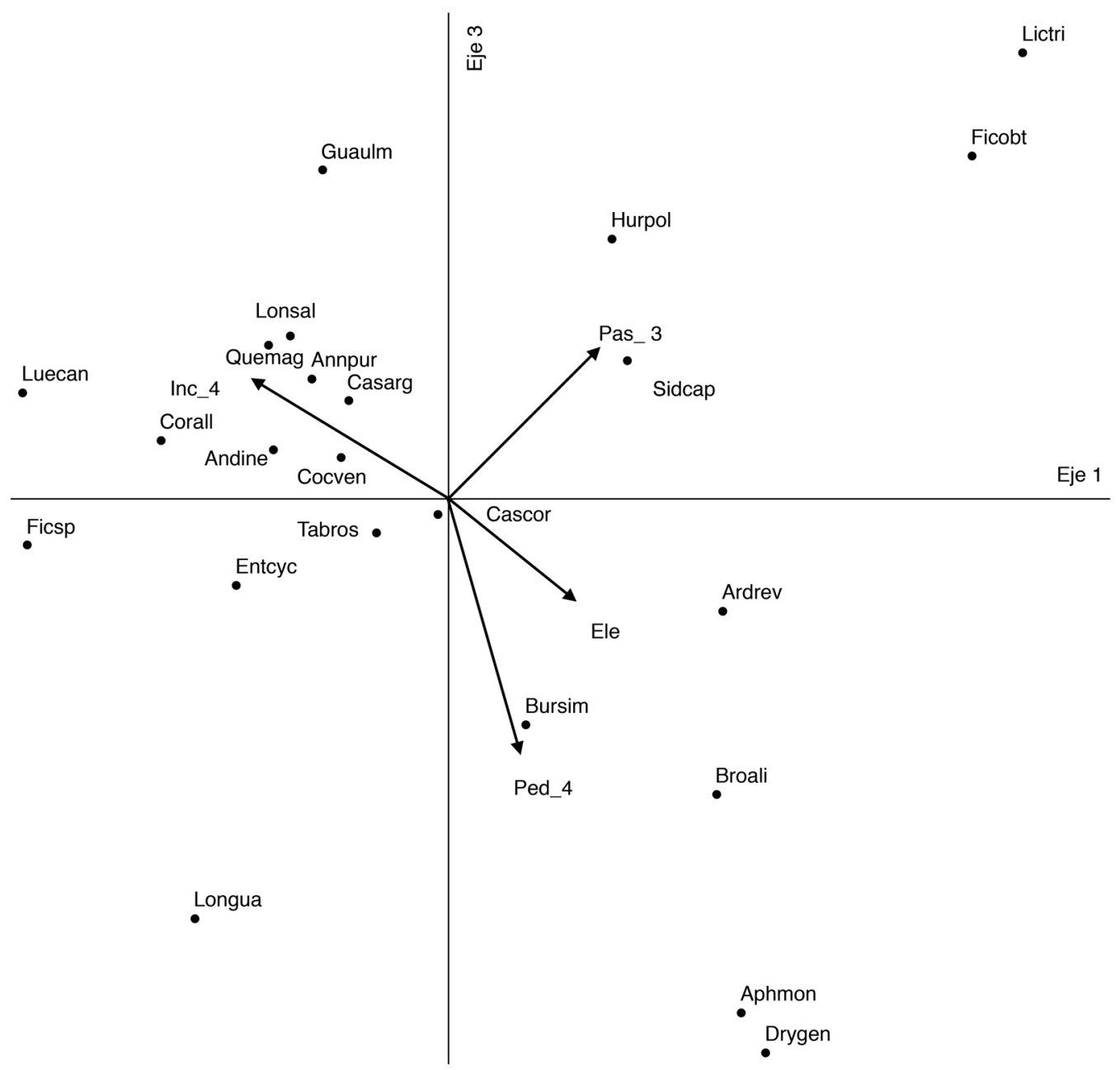

Fig. 3. Gráfico de CCA para los ejes 1 y 3. Los puntos indican las especies, para sus nombres ver pie de la Fig. 2. Los vectores representan variables ambientales, Inc_4 = incendios severos, Pas_3 $=$ pastoreo fuerte, Ele $=$ elevación, Ped_4 $=$ parcelas con el $60-80 \%$ de su superficie cubierta por piedras.

Fig. 3. CCA graph for axes 1 and 3. The dots indicate the species, for their names see figure 2. Vectors represent environmental variables, Inc $4=$ severe fires, Pas_3 = strong grazing, Ele = elevation, Ped-4 = plots with $60-80 \%$ of their area covered by stones.

$\mathrm{y}$ de las variables ambientales una $\mathrm{p}=0.005$. De acuerdo con el diagrama de ordenación, $A$. purpurea y las especies con las que tiene mayor relación, presentaron sus mayores IVI en los sitios con incendios severos (Inc_4), más precipitación anual (Pan), menor elevación y con menos pedregosidad (Fig. 3). En general fueron también estas variables las que presentaron sus mayores correlaciones con los ejes (en el sentido de ter Braak, 1986) (Tabla 3).

\section{DISCUSIÓN}

Estructura poblacional de $A$. purpurea y su relación con variables ambientales: $\mathrm{La}$ altura promedio de $A$. purpurea en el occidente de México, se encuentra dentro de las registradas para la especie; pero aquellas hasta de 18 $\mathrm{m}$, son superiores a las que se mencionan en las descripciones de la especie (Standley, 1920; Standley \& Steyermark, 1946; Schatz, 2009). 
TABLA 3

Coeficientes canónicos obtenidos a través de la regresión múltiple con CCA, correlaciones de las variables ambientales con los ejes y correlaciones de A. purpurea y otras especies con los ejes

TABLE 3

Canonical coefficients getted through multiple regression with CCA, correlations of the environmental variables with the axes and correlations of $A$. purpurea and other species with the axes

\begin{tabular}{lcccccc} 
& \multicolumn{3}{c}{ Coeficientes estandarizados } & \multicolumn{3}{c}{ Correlaciones con los ejes } \\
& Vje 1 & Eje 2 & Eje 3 & Eje 1 & Eje 2 & Eje 3 \\
Elevación & 0.332 & 1.145 & 0.029 & 0.419 & 0.763 & -0.311 \\
Profundidad de horizonte superficial & -0.188 & -0.016 & -0.241 & 0.537 & -0.164 & 0.119 \\
Tocones & 0.048 & 0.080 & 0.017 & 0.113 & 0.043 & -0.085 \\
Precipitación anual & 0.178 & 0.321 & 0.382 & -0.414 & 0.543 & 0.056 \\
Pedregosidad (Ped_2). & -0.307 & -0.595 & -0.738 & -0.278 & 0.288 & 0.173 \\
Pedregosidad (Ped_3). & -0.115 & 0.061 & -0.110 & -0.073 & 0.136 & 0.083 \\
Pedregosidad (Ped_4). & -0.130 & -0.281 & -0.704 & 0.241 & -0.038 & -0.773 \\
Pastoreo fuerte (Pas_3). & 0.427 & -0.228 & 0.250 & 0.493 & -0.063 & 0.461 \\
Pastoreo severo (Pas_4). & -0.137 & -0.257 & -0.627 & -0.424 & -0.040 & -0.520 \\
Incendios ligeros (Inc_1). & -0.948 & 0.211 & -0.368 & -0.098 & 0.242 & -0.163 \\
Incendios severos (Inc_4). & -0.928 & 0.638 & 0.300 & -0.653 & 0.035 & 0.366 \\
Annona purpurea & & & & -0.451 & 0.449 & 0.363 \\
Casearia arguta & & & & -0.330 & -0.274 & 0.298 \\
Enterolobium cyclocarpum & & & & -0.703 & 0.676 & -0.262 \\
Guazuma ulmifolia & & & & -0.418 & 0.444 & 1.00 \\
Quercus magnoliifolia & & & & -0.596 & 0.637 & 0.470 \\
Tabebuia rosea & & & -0.239 & 0.233 & -0.104 \\
\hline
\end{tabular}

Ped $\_=20-40 \%$ superficie de la parcela cubierta de piedras, Ped $3=40-60 \%$ superficie con piedras, Ped $4=60-80 \%$ superficie con piedras.

Ped_2 $=20-40 \%$ area of the plot covered with stones, Ped_3 $=40-60 \%$ area with stones, Ped_4 $=60-80 \%$ area with stones.

Esto podría interpretarse de que en la región de estudio, existen condiciones ambientales que permiten un mejor desarrollo en la altura de la especie o bien que en otras localidades no se han hecho mediciones más precisas de su altura y se han empleado las que se registran en la literatura. No se encontró información para comparar la ramificación de la especie por debajo de $1.30 \mathrm{~m}$ de altura. El que se registren individuos con más de 10 tallos, pudiera atribuirse a la capacidad de generar brotes que tiene la especie, lo cual ya ha sido registrado en otras investigaciones (Griscom, Griscom, \& Ashton, 2009). Los porcentajes altos de densidad de tallos, AB e IVI de A. purpurea, es coincidente con lo registrado en otros estudios cercanos al área de estudio (Cuevas, 2002).
La estructura diamétrica de la población en forma de "J" invertida, indica una buena reincorporación de individuos (Bongers, Pompa, Meave del Castillo, \& Carabias, 1988), aunque ha sido registrado más para especies umbrófilas (Cuevas, López-Mata, \& García-Moya, 2002). Es de considerar que los histogramas pueden diferir en función del criterio que se siga para generar el número y la amplitud de clases diamétricas en la población, (Fox, 2016). Si aplicáramos el criterio de Hubbell y Foster (1987), clases de tamaño que consecuentemente duplican a la que le precede, la población de $A$. purpurea (gráfico no presentado), se ajusta más a las especies heliófilas o pioneras consignadas en ese trabajo (Hubbell \& Foster, 1987). Las densidades grandes de tallos en las primeras 
categorías diamétricas, se atribuye a la capacidad que tiene la especie para regenerar en claros grandes, los cuales son comunes y continuos en la región de estudio, lo que podría promover que la especie colonice y se establezca en forma continua en área abiertas (obs. de los autores en campo), a pesar de su escasa o nula regeneración bajo su propio dosel. La relación positiva de la regeneración con la incidencia de Inc, es un elemento más de apoyo de que la especie requiere de espacios abiertos para su establecimiento, donde la semilla pueda llegar por dispersores, principalmente mamíferos medianos, entre los que se han observado en campo al mapache, zorra, coyote, tejón y jabalí, algunos de los cuales ya han sido registrados como dispersores de semillas de Annona (Natural Heritage Trust, 2003; Medrano-Nájera, Ramírez-Pinero, Guevara-Sada, 2014). Otros elementos que dan soporte para considerar a A. purpurea como una especie con preferencia por áreas abiertas, en bosque tropical subcaducifolio, son caracteres que tiene la especie y que han sido considerados de taxones pioneros (Swaine \& Whitmore, 1988). Como ejemplo, el tener hojas caducifolias, membranáceas y grandes (Standley, 1920; Standley \& Steyermark, 1946; Schatz, 2009; Topete-Corona, 2018), multiplicación de tallos por brotes, escasa repoblación de plántulas y juveniles bajo su dosel (en este trabajo).

Las correlaciones positivas del IVI con la Tma y el $A B$ e IVI con la incidencia de incendios, son elementos que apoyan de que A. purpurea es una especie de zonas tropicales cálidas, donde hay perturbaciones continuas que generan claros grandes (Denslow, 1980; Hubbell et al., 1999). Esto último también soportado por el que la mayor densidad de tallos se encuentra en donde hay más tocones y por consiguiente mayor apertura del dosel. Las aseveraciones de que, en algunas regiones de México, las poblaciones de A. purpurea están disminuyendo debido principalmente a la alteración de su hábitat (Vidal-Lezama et al., 2019), parecen no aplicar para el occidente de México, donde actividades como la apertura de áreas para la ganadería y la incidencia de incendios favorecen a la especie.

\section{Estructura de la comunidad y hábitat} de $A$. purpurea: La riqueza de familias, géneros y especies registradas en 1.2 ha, está por debajo de lo encontrado en superficies iguales en bosques tropicales subcaducifolios cercanos (Vázquez-Magaña \& Mendoza-Cortes, 2006), diferencias que podrían atribuirse a que son áreas con diferentes grados de conservación. La riqueza de taxones es superior a los registrado en áreas de la costa del estado de Jalisco (González-Cueva, Aguirre-Calderón, AlanísRodríguez, \& Corral-Rivas, 2018), diferencias que podrían atribuirse al límite inferior de los diámetros de los tallos que fueron considerados en las investigaciones, dap $\geq 2.5 \mathrm{~cm}$ en el primero y dap $>7.5 \mathrm{~cm}$ en el segundo. Las familias y géneros registrados con mayor riqueza específica, también coinciden con lo encontrado por Cuevas (2002), en lo que llamó bosque tropical subcaducifolio de partes bajas. Aunque puede haber coincidencia, con otras investigaciones, con algunas de las familias con mayor riqueza en este tipo de vegetación, no ocurre lo mismo con los géneros y las especies con las mayores abundancias, AB e IVI, lo cual se atribuye a que cuando se cambia de una localidad a otra, sobre todo cuando las distancias entre los sitios que se comparan se incrementan, las condiciones ambientales varían y los estados de conservación o sucesión son diferentes (Gutiérrez-Báez, Zamora-Crescencio, \& Hernández-Mundo, 2012; Dzib-Castillo, Chanatásig-Vaca, \& González-Valdivia, 2014; González-Cuevas et al., 2018).

Las especies con las que $A$. purpurea comparte los mayores índices de asociación como G. ulmifolia, T. rosea, C. arguta, E. cyclocarpum, han sido registradas como sus compañeras en la región (Cuevas, 2002; Vázquez-Magaña \& Mendoza-Cortés, 2006), pero también en Centro y Sudamérica (Oviedo-Pérez, 2015; Cabrera-Amaya \& Rivera-Díaz, 2016), sobre todo la asociación de A. purpurea y G. ulmifolia, grupo que fue estadísticamente diferente a los restantes (Fig. 2). Esta asociación podría 
atribuirse a que las dos especies tienen requerimientos ambientales similares, sobre todo el hecho que se ven favorecidas en áreas de vegetación secundaria derivada de bosques tropicales húmedos, y tienen en común una fuerte capacidad de multiplicación por rebrote (Griscom et al., 2009).

Las variables ambientales recuperadas por el CCA como de más influencia sobre la distribución y abundancia de $A$. purpurea y de las especies con las que presenta mayores asociaciones (G. ulmifolia, Q. magnoliifolia, E. cyclocarpum), son la Pan, los incendios severos (Inc_4), menor Ele y menos Ped (Fig. 3, Tabla 3). La influencia de la Pan en este grupo de especies, podría ser interpretada como la preferencia de ellas por los lugares más húmedos. Annona purpurea tiene algunas características morfológicas y fisiológicas que parecen responder a esta variable, como el poseer hojas membranáceas y grandes, las cuales generan periodos de crecimiento estacional, aprovechando la humedad durante el periodo corto de lluvias, con abscisión del follaje en la estación seca y más larga del año (Reicht, 2014). Lo anterior permitiría explicar porque los mayores atributos estructurales de A. purpurea se registran en los bosques más húmedos (bosque tropical subcaducifolio), aunque algunas veces se pueden registrar individuos en el bosque tropical caducifolio o en la transición con el bosque de encino. La influencia de la precipitación sobre $A$. purpurea ya ha sido registrada y se ha encontrado un acoplamiento de la producción de hojas y flores con el inicio de las lluvias y la maduración de los frutos con el final de la temporada (González-Esquinca, De la Cruz, Castro-Moreno, Riley-Saldaña, 2016). El grupo de especies mencionado también presenta una relación positiva y estrecha con Inc_4, lo que podría atribuirse a que se trata de especies que requieren apertura de claros grandes para su establecimiento y que se ven favorecidas en lugares con perturbaciones, incluyendo el fuego (López-Moctezuma, Rodríguez-Trejo, Santiago-Cortes, \& Granado-Sánchez 2015; Oviedo-Pérez, 2015; CONABIO, 2020; IFOCATIE, 2020). Otra variable importante fue la Ele, registrando $A$. purpurea sus mayores valores estructurales en los sitios con menor elevación, si consideramos que esta variable estuvo correlacionada en forma negativa con la Tma, entonces existe una preferencia de la especie por los lugares más cálidos, lo cual coincide con lo registrado por Cuevas (2002) y Vázquez-Magaña y Mendoza-Cortés (2006) (Fig. 3, Tabla 3).

Concluimos que $A$. purpurea presenta una estructura poblacional con buena reincorporación de individuos y que su distribución y abundancia parecen responder principalmente a factores ambientales como precipitación anual, temperatura media anual, pedregosidad y a perturbaciones causadas por incendios y ganadería.

Declaración de ética: los autores declaran que todos están de acuerdo con esta publicación y que han hecho aportes que justifican su autoría; que no hay conflicto de interés de ningún tipo; y que han cumplido con todos los requisitos y procedimientos éticos y legales pertinentes. Todas las fuentes de financiamiento se detallan plena y claramente en la sección de agradecimientos. El respectivo documento legal firmado se encuentra en los archivos de la revista.

\section{AGRADECIMIENTOS}

El primer autor recibió una beca del Consejo Nacional de Ciencia y Tecnología para cursar una Maestría en Ciencias en la Universidad de Guadalajara. El trabajo de campo fue financiado por la Universidad de Guadalajara y la Secretaría de Educación Pública. Personal del Herbario ZEA apoyo en el trabajo de campo y en la identificación de material botánico. Enrique Jardel proporcionó información climática sobre los sitios de muestreo.

\section{RESUMEN}

Introducción: Annona purpurea es un árbol Mesoamericano, distribuido por la vertiente pacífica y atlántica de México hasta Sudamérica. Diferentes partes de la planta son de utilidad y los frutos son comestibles. 
Objetivo: Describir la estructura poblacional, el hábitat y los factores ambientales que influyen en la distribución y abundancia de $A$. purpurea en el occidente de México. Métodos: De abril a noviembre 2015, se establecieron 24 unidades de muestreo de $500 \mathrm{~m}^{2}$ cada una, con presencia de A. purpurea. En cada sitio se midieron e identificaron todas las especies leñosas con diámetro a la altura de pecho (dap) $\geq 2.5 \mathrm{~cm}$ y se registró información ambiental, geográfica y climática. Se obtuvo información sobre la repoblación de la especie. Se estimó la estructura de $A$. purpurea y se examinaron sus relaciones con las variables ambientales. El índice de asociación de Whittaker se determinó utilizando la matriz de índice de valor de importancia (IVI). Con la matriz de variables ambientales y la de IVI, se realizó un Análisis de Correspondencia Canónica para determinar la influencia de las variables ambientales sobre la especie y visualizar la distribución de las especies en el espacio multidimensional. Resultados: A. purpurea presentó 1108 tallos en 1.2 ha, con el $85 \%$ de ellos concentrados en las tres primeras categorías diamétricas. Su densidad presentó una relación positiva con la presencia de tocones, mientras que el área basal y el IVI se relacionaron de manera similar con la incidencia de incendios. Hubo poca o nula regeneración de $A$. purpurea debajo de su dosel. Los mayores IVI en la comunidad se registraron para A. purpurea, Tabebuia rosea, Quercus magnoliifolia y Enterolobium cyclocarpum y la prueba de perfiles de semejanza separó a A. purpurea y Guazuma ulmifolia como un grupo diferente. Las variables ambientales con mayor influencia en la distribución y abundancia de $A$. purpurea fueron la precipitación anual, incidencia de incendios, elevación, temperatura media anual, pedregosidad y pastoreo. Conclusión: A. purpurea presenta los mayores atributos estructurales en la comunidad, sus poblaciones se ven favorecidas en sitios con mayor precipitación y temperatura, con poca pedregosidad, donde hay incidencia de perturbaciones por fuego y ganado que generan claros grandes y su mayor asociación la presenta con G. ulmifolia.

Palabras claves: bosque tropical subcaducifolio; cabeza de negro; fuego; ganadería; vegetación secundaria.

\section{REFERENCIAS}

Andres-Agustín, J. (2015). Situación actual de las investigaciones de las anonáceas en México. Recursos fitogenéticos de las anonáceas en el estado de Veracruz. En E. Vidal-Lezama, N.A. Vidal-Martínez, \& L. Vidal-Hernández (Eds.), Anonáceas. Plantas antiguas. Estudios recientes (Parte 2, pp. 27-39). Chapingo, Texcoco, México: Universidad Autónoma Chapingo.

Balzarini, M.G., Gonzalez, L., Tablada, M., Casanoves, F., Di Rienzo, J.A., \& Robledo, C.W. (2008). InfoStat Manual del Usuario. Córdoba, Argentina: Editorial Brujas.
Beers, T.W., Dress, P.E., \& Wensel, L.C. (1966). Aspect transformation in site productivity research. Journal of Forestry, 64, 691-692.

Bongers, F., Pompa, J., Meave del Castillo, J., \& Carabias, J. (1988). Structure and floristic composition of the lowland rain forest of Los Tuxtlas, México. Vegetatio, 74, 55-80. DOI: 10.1007/bf00045614

Cabrera-Amaya, D.M., \& Rivera-Díaz, O. (2016). Composición florística y estructura de los bosques ribereños de la cuenca baja del río Pauto, Casanare, Colombia. Caldasia, 38(1), 53-85. DOI: 10.15446/caldasia. v38n1.57829

Clarke, K.R., Gorley, R.N., Somerfield, P.J., \& Warwick, R.M. (2014). Change in marine communities: an approach to statistical analysis and interpretation ( $3^{\text {era }}$ edición). Albany, New Zealand: PRIMER-E Ltd.

Clarke, K.R., \& Gorley, R.N. (2015). PRIMER v7: User Manual/Tutorial. Plymouth, Engand: PRIMER-E.

Comisión Nacional para el Conocimiento y Uso de la Biodiversidad (CONABIO). (2020). Tabebuia rosea y Enterolobium cyclocarpum. Recuperado de http:// www.conabio.gob.mx/conocimiento/info_especies/ arboles/doctos/indice_especies.html

Cuevas, G.R. (2002). Análisis de gradientes de la vegetación de la cañada El Tecolote, en la sierra de Manantlán, Jalisco, México (Tesis doctoral). Colegio de Posgraduados, Montecillo, Texcoco, México.

Cuevas, G.R., López-Mata, L., \& García-Moya, E. (2002). Primer registro de Desmopsis trunciflora (Schlecht. \& Cham) G.E. Schatz (Annonaceae) para el occidente de México y análisis de su población en la sierra de Manantlán, Jalisco. Acta Botanica Mexicana, 58, 7-18. DOI: 10.21829/abm58.2002.887

Cuevas, G.R., García-Moya, E., Vázquez-García, J.A., \& Núñez-López, N.M. (2008). Estructura poblacional y relaciones ambientales del árbol tropical Nectandra rudis (Lauraceae), una especie rara en el occidente de México. Revista de Biología Tropical, 56, 247-256. DOI: $10.15517 /$ rbt.v56i1.5521

Curtis, J.T., \& McIntosh, R.P. (1951). An upland forest continuum in the praire forest border region of Wisconsin. Ecology, 32, 476-496. DOI: 10.2307/1931725

De la Cruz, I., Castro-Montero, M., \& González-Esquinca, A.R. (2016). La familia Annonaceae Juss., en México. Lacandonia, 10(2), 71-82.

Denslow, J.S. (1980). Gap partitioning among tropical rainforest trees. Biotropica, 12(2), 47-55. DOI: $10.2307 / 2388156$

Di Rienzo, J.A., Casanoves, F., Balzarini, M.G., Gonzalez L., Tablada, M., \& Robledo, C.W. (2019). InfoStat, versión 2019, Grupo InfoStat, FCA, Universidad Nacional de Córdoba, Argentina. 
Dzib-Castillo, B., Chanatásig-Vaca, C., \& González-Valdivia, N.A. (2014). Estructura y composición en dos comunidades arbóreas de la selva baja caducifolia y mediana subcaducifolia en Campeche, México. Revista Mexicana de Biodiversidad, 85, 167-178. DOI: $10.7550 / \mathrm{rmb} .38706$

Fernández, E.A., Zavala, H.J., \& Romero, C.R. (2010). Digital Climatic Atlas of México. 3rd International Conference on Cartography and GIS. June, 2010, Nessebar, Bulgaria. Recuperado de http://www.cartography-gis.com/pdf/44_Fernandez_Eguiarte_Mexico_paper.pdf

Fox, J. (2016). Applied regression analysis and generalized linear models ( $3^{\text {era }}$ edición). Los Ángeles, California, EEUU: Publicaciones SAGE, Inc.

González-Cueva, G.A., Aguirre-Calderón, O.A., AlanísRodríguez, E., \& Corral-Rivas, J.J. (2018). Estructura y composición florística de un bosque tropical subcaducifolio en Jalisco, México. e-CUCBA, 9, 9-16.

González-Esquinca, A.R, De la Cruz, I., Castro-Moreno, M., \& Riley-Saldaña, C.N. (2016). Phenological strategies of Annona species from the tropical deciduos forest of Chiapas, Mexico. Botanical Sciences, 94(3), 531-541. DOI: 10.17129/botsci.645

Griscom, H.P., Griscom, B.W., \& Ashton, M.S. (2009). Forest regeneration from pasture in the dry tropics of Panama: effects of cattle, exotic grass, and forested riparia. Restoration Ecology, 17(1), 117-126. DOI: 10.1111/j.1526-100X.2007.00342.x

Gutiérrez-Báez, C., Zamora-Crescencio, P., \& Hernández-Mundo, S.C. (2012). Estructura y composición florística de la selva mediana subcaducifolia de Mucuychacán, Campeche, México. Foresta Veracruzana, 14(1), 9-16.

Hubbell, S.P., \& Foster, R.B. (1987). La estructura espacial en gran escala de un bosque neotropical. En D.A. Clark, R. Dirzo, \& N. Fetcher (Eds.), Ecología y ecofisiología de plantas en los bosques mesoamericanos. Revista de Biología Tropical(Supl. 1), 7-22.

Hubbell, S.P., Foster, R.B., O’Brien, S.T., Harms, K.E., Condit, B., Wechsler, B., ... Lao de Lao, S. (1999). Light-gap disturbances, recruitment limitation, and tree diversity in a neotropical forest. Science, 283, 554-557. DOI: 10.1126/science.283.5401.554

Instituto Forestal de Oxford y Centro Agronómico Tropical de Investigaciones y Enseñanza (IFO-CATIE). (2020). Guazuma ulmifolia. Recuperado de www. arbolesdecentroamerica.info

Jiménez-Ramírez, J., \& Soto-Núñez, J.C. (2015). Annona contrerasii (Annonaceae), especie nueva del estado de Guerrero, México. Acta Botanica Mexicana, 112, 37-43. DOI: 10.21829/abm112.2015.1087
López-Moctezuma, M.A., Rodríguez-Trejo, D.A., Santiago-Cortes, F., \& Granado-Sánchez, D. (2015). Tolerancia al fuego en Quercus magnoliifolia. Arvore, Viçosa-MG, 39(3), 523-533. DOI: 10.1590/0100-67622015000300013

Luna-Cázares, L.M., \& González-Esquinca, A.R. (2008). Actividad antibacteriana de extractos de Annona diversifolia Safford y Annona purpurea Mociño \& Sessé ex Dunal. Polibotánica, 25, 121-125.

Luna-Cázares, L.M., \& González-Esquinca, A.R. (2015). La chinguya (Annona purpurea Moc. \& Sessé ex Dunal): una planta Mesoamericana. Recursos fitogenéticos de las anonáceas en el estado de Veracruz. En E. Vidal-Lezama, N.A. Vidal-Martínez, \& L. VidalHernández (Eds.), Anonáceas. Plantas antiguas. Estudios recientes (Parte 2, pp. 229-245). Chapingo, Texcoco, México: Universidad Autónoma Chapingo.

Mass, P.J.M., Westra, L.Y.T., Chatrou, L.W., Verspagen, N., Rainer, H., Zamora, N.A., \& Erkens, R.H.J. (2019). Twelve new and exciting Annonaceae from the Neotropics. PhytoKey, 126, 25-69. DOI: 10.3897/ phytokeys.126.33913

McCune, B., \& Grace, J.B. (2002). Analysis of ecological communities. Oregon, EEUU: MjM Software Design.

McCune, B., \& Mefford, M.J. (2011). PC-ORD. Multivariate analysis of ecological data. Version 6.0. Oregon, EEUU: MjM Software Design.

Medrano-Nájera, R., Ramírez-Pinero, M., \& Guevara-Sada, S. (2014). Una mirada a la dispersión de semillas en las excretas de mamíferos. Cuadernos de Biodiversidad, 46, 19-28.

Natural Heritage Trust. (2003). Pond apple (Annona glabra). Australia: Weed Management Guide.

Olvera, V.M., Moreno, G.S., \& Figueroa, R.B.L. (1996). Sitios permanentes para la investigación silvícola. Guadalajara, Jalisco, México: Universidad de Guadalajara.

Oviedo-Pérez, P.E. (2015). Composición y estructura forestal de la Reserva Absoluta de Cabo Blanco y remanentes de bosque del sector de Cabuya, Puntarenas, Costa Rica. Repertorio Científico, 18(2), 95-102.

Reich, P.B. (2014). The world-wide 'fast-slow' plant economics spectrum: a traits manifesto. Journal of Ecology, 102, 275-301. DOI: 10.1111/1365-2745.12211

Rzedowski, J., \& McVaugh, R. (1966). La vegetación de Nueva Galicia. Contributions from the University of Michigan Herbarium, 9(1), 1-123.

Schatz, G.E. (2009). Annonaceae. Flora de Nicaragua. Recuperado de legacy.tropicos.org/ name $/ 01600805$ ? proyectid $=7$ 
Standley, P.C. (1920). Trees and shrubs of Mexico. Contributions from the United Sates National Herbarium (Vol. 23). USA: United States National Museum. DOI: $10.5962 /$ bhl.title. 15726

Standley, P.C., \& Steyermark, J.A. (1946). Annonaceae. Flora de Guatemala. Fieldiana: Botany, 24(4), 270-294.

Swaine, M.D., \& Whitmore, T.C. (1988). On the definition of ecological species groups in tropical forest. Vegetatio, 75 , 81-86. DOI: 10.1007/bf00044629

ter Braak, C.J.F. (1986). Canonical correspondence analysis: a new eigenvector technique for multivariate direct gradient analysis. Ecology, 67, 1167-1179.

Topete-Corona, C. (2018). Ecología de Annona purpurea (Annonaceae) y su importancia económica en el occidente de México (Tesis de Maestría). Universidad de Guadalajara, Autlán de Navarro,
Jalisco, México. Recuperado de https://riudg.udg.mx/ handle/20.500.12104/80303

Vázquez-García, J.A., \& Givnish, T. (1998). Altitudinal gradient in a tropical forest composition, structure, and diversity in the Sierra de Manantlan. Journal of Ecology, 86, 999-1020. DOI: 10.1046/j.1365-2745.1998.00325.x

Vázquez-Magaña, M.Y., \& Mendoza-Cortés, J.L. (2006). Estructura, composición florística y diversidad del bosque tropical subcaducifolio en tres localidades de la costa sur de Jalisco (Tesis). Universidad de Guadalajara, Autlán de Navarro, Jalisco, México. https:// riudg.udg.mx/handle/20.500.12104/73422

Vidal-Lezama, E., Villegas-Monter, A., Vaquera-Huerta, H., Robledo-Paz, A., \& Martínez-Palacios, A. (2019). Annona purpurea Moc. \& Sessé ex Dunal native species of México, underutilized. Agroproductividad, 12(3), 9-15. DOI: 10.32854/agrop.v0i0.1326 